\title{
Grinding Burn Detection Based on Cross Wavelet and Wavelet Coherence Analysis by Acoustic Emission Signal
}

\author{
Zheyu Gao ${ }^{1}$, Jing Lin² ${ }^{2}$, Xiufeng Wang ${ }^{1}$ and Yuhe Liao ${ }^{1}$
}

\begin{abstract}
Grinding burn monitoring is of great importance to guarantee the surface integrity of the workpiece. Existing methods monitor overall signal variation. However, the signals produced by metal burn are always weak. Therefore, the detection rate of grinding burn still needs to be improved. The paper presents a novel grinding burn detection method basing on acoustic emission (AE) signals. It is achieved by establishing the coherence relationship of pure metal burn and grinding burn signals. Firstly, laser and grinding experiments were carried out to produce pure metal burn signals and grinding burn signals. No-burn and burn surfaces were generated and AE signals were captured separately. Then, the cross wavelet transform (XWT) and wavelet coherence (WTC) were applied to reveal the coherence relationship of the pure metal burn signal and grinding burn signal. The methods can reduce unwanted AE sources and background noise. Novel parameters based on XWT and WTC are proposed to quantify the degree of coherence and monitor the grinding burn. The grinding burn signals were recognized successfully by the proposed indexes under same grinding condition.
\end{abstract}

Keywords: Grinding burn, Cross wavelet transform, Wavelet coherence, Acoustic emission

\section{Introduction}

Grinding burn is caused by excessive heat produced during grinding process. It changes the surface hardness and reduces the fatigue resistance which brings great damage to work-piece $[1,2]$. Grinding burn is widespread in the field of cylindrical grinding, rail grinding, bearing rings grinding, and so on [3-5]. Therefore, the detection and monitoring of grinding burn is of great importance to guarantee the high quality of mechanical components.

Various monitoring methods basing on vibration [6], electric current [7], force [8], piezoelectric diaphragms [9], acoustic emission (AE) [10], Barkhausen [11], eddy current [12], and so on [13-15] are utilized to detect the occurrence of grinding burn. Among all these on line detection methods, $\mathrm{AE}$ is one of the most effective technology for grinding burn detection [7]. Besides, AE senor

\footnotetext{
*Correspondence: linjing@buaa.edu.cn

${ }^{2}$ School of Reliability and Systems Engineering, Beihang University, Beijing 100191, China

Full list of author information is available at the end of the article
}

is easy to install and sensitive to the grinding signals [16]. Hence, it has been widely studied to determine the grinding burn occurrence.

Lots of experiments concerned with grinding monitoring were carried out. Jemielniak et al. [17] studied the relationship between wear of tool and grinding burn by examining the tool surface and AE signals [17]. Aguiar et al. [7] did the grinding experiment, during which $\mathrm{AE}$ signals and parameters were calculated to indicate the grinding burn [7]. Bell et al. [18] developed the grinding temperature prediction diagrams in high deep grinding process [18]. Griffin et al. [19] established the relationship of micro unit grit events of AE signals and macro phenomena during grinding burn process [19]. Researchers simulated the grinding burn process by laser, from which pure AE burn signals were obtained. The results were used as reference to identify grinding burn [20-22]. Wu et al. [23] carried out a series of grinding experiments to monitor grinding burn by $\mathrm{AE}$ sensor, dynamometer and surface roughness [23]. 
A plenty of signal processing methods were adopted to study the grinding burn detection. Parameters of AE signals such as root mean square (RMS), mean-value deviance (MVD), constant false alarm rate (CFAR) etc. have been calculated and compared [7]. Then time-frequency methods such as Short Time Fourier Transform (STFT) [22], Wavelet Packet Transform (WPT) [20], Hilbert Huang Transform (HHT) [24] and Ensemble Empirical Mode Decomposition (EEMD) [21] have been introduced to analyze grinding burn signals.

However, the detection rate of grinding burn still needs to be improved. Hence, a great deal of intelligence algorithms were employed to increase the identification accuracy. Liu et al. [25] used Fuzzy Pattern Algorithm to predict the grinding burn [25]. Wang et al. [26] established modals and utilizes Artificial Neural Networks (ANN) to predict different degrees of grinding burn [26]. Support Vector Machine (SVM) and Principle Component Analysis (PCA) were also employed to classify the grinding burn [27, 28]. Besides, Saravanapriyan et al. [29] selected force, vibration and AE signals as input information of ANN to predict grinding burn [29].

Although lots of work has been done, there are still some key problems to be solved. Firstly, the accuracy of traditional grinding burn identification methods basing on feature parameters is still need to be improved. Novel methods like EEMD and intelligence algorithms have high detection rate. However, these methods cost much computing time. Therefore, a new grinding burn detection method which has high accuracy and costs less time is needed. Secondly, the AE signal components which can indicate grinding burn are always covered by other $\mathrm{AE}$ sources and noises of the grinding process. Hence, new method which can eliminate the undesired signals is needed. Thirdly, although laser experiments have been carried out to produce pure AE burn signals, how does the pure metal burn signals effect the grinding burn signals is unknown. The correlation relationship of the two kinds of burn signals can provide more information to detect grinding burn.

Above mentioned problems are solved in this paper. A novel detection method based on the Cross Wavelet Transform (XWT) and Wavelet Coherence (WTC) is proposed [30]. It has high accuracy and costs less time. The common area with high energy and coherence relationship of pure metal burn signals and grinding burn signals were obtained by XWT and WTC, from which most of the interference signals and noises were eliminated. Besides, the correlation relationship of two kinds of burn signals were found and quantified by energy of XWT (EXWT) and degree of WTC (DWTC). The two indexes were employed to identify normal grinding and grinding burn signals successfully.
The organization of the paper is as follows. In Section 2, the definition of XWT and WTC are introduced. Section 3 describes the laser experiment and grinding experiment. In Section 4, the pure AE burn signals and grinding burn signals are analyzed by XWT and WTC methods. The feature parameters for grinding burn detection are defined and utilized to classify no-burn and burn AE signals. Section 5 provides the conclusion.

\section{Theory}

In order to find the relationship of pure metal burn signals and grinding burn signals, signal correlation needs to be found. For the transient non-stationary AE signals, Fourier-based coherence method is unable to detect temporal structure of $\mathrm{AE}$ signals. Hence, the time-frequency coherence methods should be utilized to analyze signals. XWT exposes common areas with high power and WTC reveals the coherence of two signals in time-frequency space. They have been widely applied to find the co-vary information of geophysical signals and brain signals [31-33]. The XWT has been used to demonstrate similar variation trend of current and vibration signals [34]. Hence, the XWT and WTC are suitable to study relationship of the two kinds of burn signals.

\subsection{Theory of Wavelet Coherence}

The wavelet transform can be divided into discrete wavelet transform (DWT) and continuous wavelet transform (CWT). The DWT suits orthogonal wavelet bases, while both CWT and DWT can be used for non-orthogonal wavelet functions [35]. CWT is suitable to describe the similarity and singularity of signal [36]. Therefore, the CWT is used in the paper. According to reference [37], the Morlet wavelet is most suitable for the analysis of $\mathrm{AE}$ signal. Therefore, the Morlet wavelet is utilized here.

The CWT of a signal $x(t)$ is defined as follows [38]:

$$
W_{x}(u, s)=\frac{1}{\sqrt{s}} \int_{-\infty}^{+\infty} x(t) \psi^{*}\left(\frac{t-u}{s}\right) \mathrm{d} t,
$$

where $s$ represents the scale factor and $u$ behalves the time shift factor. Here ' $*$ ' denotes the complex conjugate. According to Ref. [18], the wavelet power is defined as $\left|W_{x}(u, s)\right|^{2}$.

Accordingly, the auto-spectrum of wavelet of signal $x(t)$ is defined in Eq. (2):

$$
W_{x, x}(u, s)=W_{x}(u, s) W_{x}^{*}(u, s) .
$$

And the definition of XWT of two signals $x(t)$ and $y(t)$ is provided as follows:

$$
W_{x, y}(u, s)=W_{x}(u, s) W_{y}^{*}(u, s),
$$

here, the corresponding cross wavelet power is defined as $\left|W_{x, y}(u, s)\right|$. 
It is obviously that the XWT is suitable to find the common power areas with large energy of two signals in time-frequency plane.

Consulting the description of coherence, the definition of wavelet coherence is defined in Eq. (4):

$$
\operatorname{WTC}(u, s)=\frac{\left|S\left(s^{-1} W_{x y}(u, s)\right)\right|}{\sqrt{S\left(s^{-1}\left|W_{x x}(u, s)\right|\right) S\left(s^{-1}\left|W_{y y}(u, s)\right|\right)}},
$$

where $S$ is the smooth function, $s$ is the scale factor and $u$ represents the time shift factor of CWT. Without the smooth function $S$, the WTC will be equal to one everywhere. However, the definition of the smooth operator is not uniform [27]. The smooth operator $S$ must keep balance between frequency resolution and significance [25]. Usually, the smooth function is carried out in time and scale domain. In this paper, the smoothing operator is defined as follows:

$$
\begin{aligned}
& S(W)=S_{\text {scale }}\left(S_{\text {time }}(W)\right), \\
& \left.S_{\text {time }}(W)\right|_{s}=\left.\left(W(s) * e^{\frac{-t^{2}}{2 s^{2}}}\right)\right|_{s},
\end{aligned}
$$

here, $t$ is the points averaged over a range of time, which is determined empirically and set to $t \in[-30,30]$.

$$
S_{\text {scale }}(W)=W * \prod(C s),
$$

here, $C$ is scale decorrelation length of wavelet which is determined empirically and set to $0.6[24,25]$. $\prod$ is the rectangle function.

According to Schwartz inequality, the result of WTC must between $[0,1]$. From the definition, it is known that WTC reveals the local correlation of two signals in timefrequency planes.

\section{Experiments Set-up}

In the grinding process, the grinding $\mathrm{AE}$ signal has a variety of $\mathrm{AE}$ resources. The metal burn $\mathrm{AE}$ signal is always buried in these resources. It is hard to judge whether the signal variation is induced by metal burn or by other grinding factors. In order to investigate the contribution of metal burn signal to grinding burn signal, a laser experiment was carried out to produce pure metal burn signals, during which the undesired AE resources were excluded. Then the grinding experiment was conducted and the grinding burn signals were obtained. The set-up of two experiments are given below.

\subsection{Laser Experiment}

In the experiment, laser machine is employed to generate metal burn, during which AE signals are collected simultaneously. Laser is provided by Chutian laser welding machine and the laser parameters are shown in Table 1 . The energy of laser is adjusted to produce noburn, and burn surfaces, which are confirmed by surface temperature and temper color.

The 1045 steel is adopted in the experiment. The PACII system collects the AE signals. The sample rate is set to $2 \mathrm{MHz}$. The amplification factor is $40 \mathrm{~dB}$. And the $\mathrm{AE}$ threshold is $30 \mathrm{~dB}$. The $\mathrm{AE}$ sensor, Nano30 is employed. The operating frequency range of Nano30 is between $125 \mathrm{kHz}$ to $750 \mathrm{kHz}$. And its resonant frequency is $300 \mathrm{kHz}$. The surface temperature is recorded by infrared thermal imager (FLIR-SC7000). Figure 1 shows the experimental apparatus.

\subsection{Grinding Experiment}

Grinding burn can be produced by adjusting sharpness of grinding wheel. No-burn and burn surfaces are produced by grinding machine $(\mathrm{M} 7130 \mathrm{H})$. Table 2 shows the operation parameters of grinding process. Except for the cooling condition, the grinding parameters of the experiment are same with the parameters of the real grinding process. The burn surfaces are confirmed by temper color. The AE signals are picked up during the grinding process. The $\mathrm{AE}$ acquisition system and material of workpiece are same as those used in the laser experiment. The sample rate of the grinding experiment is set to $2 \mathrm{MHz}$. The amplification factor is $40 \mathrm{~dB}$ and the threshold is $30 \mathrm{~dB}$. The schematic diagram of the experiment is shown in Figure 2.

The optical image of laser burn surface and grinding burn surface are presented in Figure 3. It can be seen that the temper color of grinding burn surface is similar to laser burn surface. No-burn surfaces have no obvious change. While burn surfaces are black and yellow. The results of two experiments are discussed in the next section.

\section{Results and Discussion}

\subsection{Result of Laser Burn Experiment}

Figure 4 is the waveforms and spectrums of $\mathrm{AE}$ signals produced by laser. Figure 4(a) represents the waveforms of no-burn and burn signal. Figure $4(\mathrm{~b})$ is the spectrums of no-burn and burn signal by laser heating. The amplitude of no-burn signal is much smaller than burn signal. The main frequencies of metal burn signal focus

Table 1 Laser parameters

\begin{tabular}{lll}
\hline Burn degrees & No-burn & Burn \\
\hline Laser energy & $5 \mathrm{~ms}-100 \mathrm{~A}$ & $5 \mathrm{~ms}-160 \mathrm{~A}$ \\
Focal distance & $123 \mathrm{~mm}$ & $123 \mathrm{~mm}$ \\
Surface temperature & $<200^{\circ} \mathrm{C}$ & $>700^{\circ} \mathrm{C}$ \\
\hline
\end{tabular}




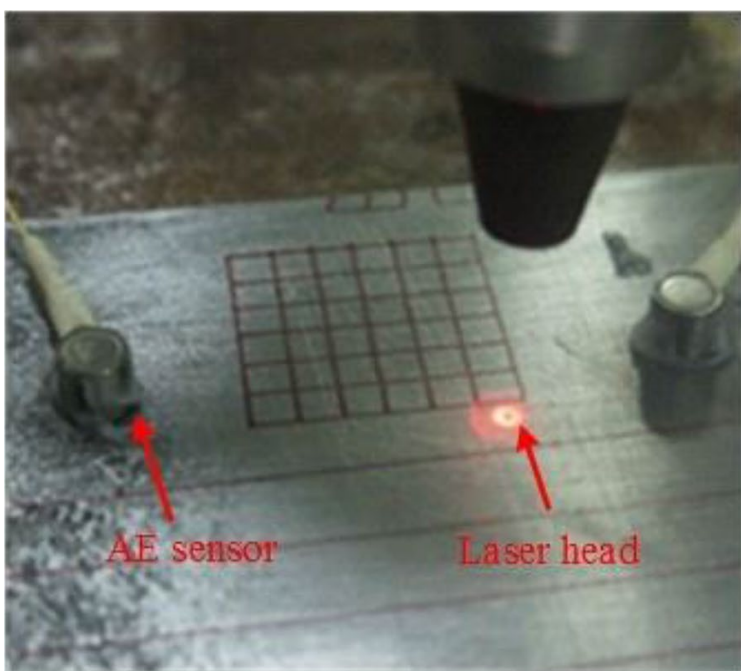

a Laser spot

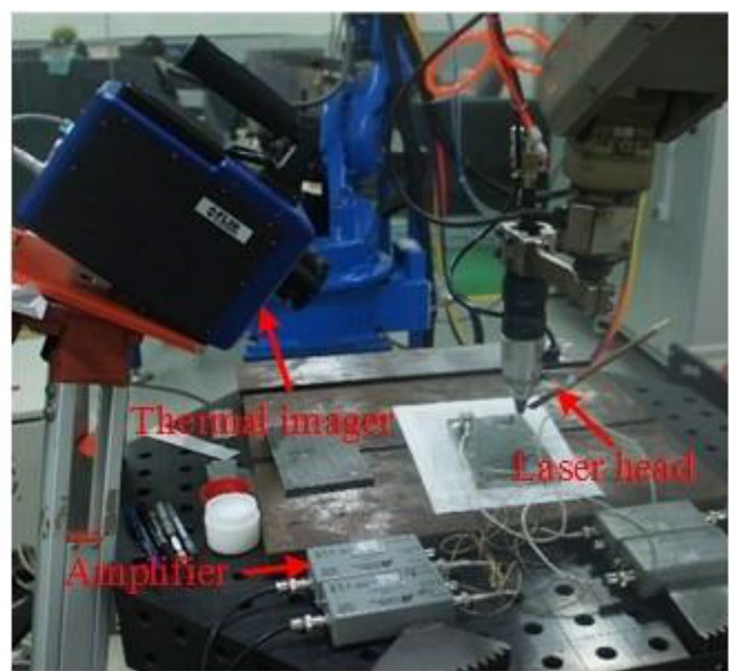

b Thermal imager and AE system

Figure 1 Laser experimental setup

\section{Table 2 Grinding parameters and different degrees of grinding burn}

\begin{tabular}{lll}
\hline Burn degrees & No-burn & Burn \\
\hline Wheel speed $(r / m i n)$ & 1440 & 1440 \\
Grinding depth $(\mu \mathrm{m})$ & 20 & 20 \\
Feed rate $(\mathrm{m} / \mathrm{min})$ & $3-27$ & $3-27$ \\
Coolant & No & No \\
\hline
\end{tabular}

in frequency band of $250 \mathrm{kHz}$ to $400 \mathrm{kHz}$. In the bandwidth of $420 \mathrm{kHz}$ to $530 \mathrm{kHz}$, the amplitude of spectrum of burn signal is still higher than the no-burn signal. Besides, between the frequency band of $600 \mathrm{kHz}$

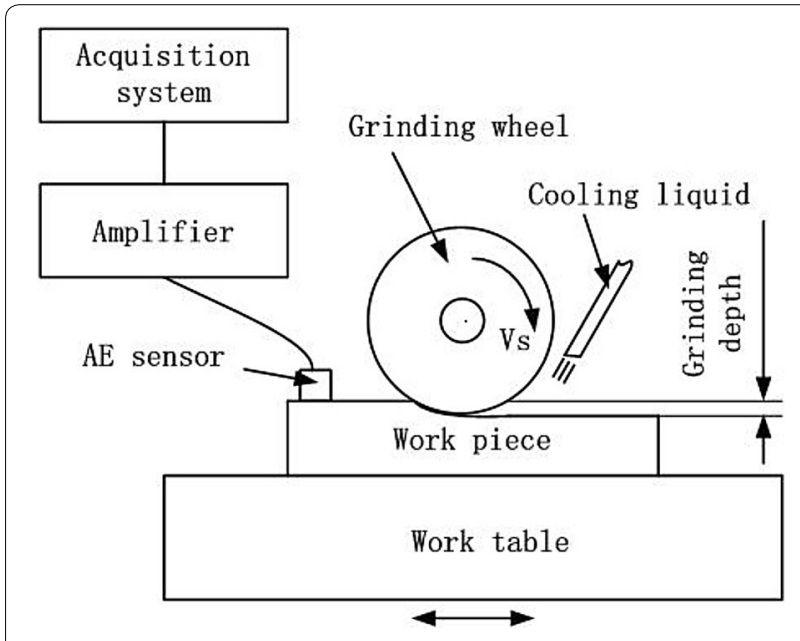

Figure 2 Schematic diagram of grinding experiment to $650 \mathrm{kHz}$, some frequency components of burn signal are slightly higher than the no-burn signal. These characteristics of frequency distribution can be utilized in further detection work.

\subsection{Result of Grinding Burn Experiment}

Figure 5 depicts the waveforms and spectrums of grinding signals. The no-burn and burn surfaces are produced by different bluntness of grinding wheel under same grinding parameters. The cooling liquid is forbidden to promote the occurrence of grinding burn. Figure 5(a) shows waveforms of the no-burn and burn signals. In order to ensure the effectiveness of the comparison, the grinding signals were truncated to the same length. Figure 5(b) presents the spectrums of no-burn and burn signals of grinding process. In Figure 5(b), the main energy of no-burn and burn signals are all below $100 \mathrm{kHz}$. The peak value of no-burn signal is even higher than burn signal. Hence it is hard to distinguish no-burn and burn signals by ordinary characteristic parameters within this bandwidth. Between frequency band of $200 \mathrm{kHz}$ to $650 \mathrm{kHz}$ which we concern, the frequency distributions of no-burn and burn signals are similar. Amplitudes of some frequency components of burn signal is slightly higher than no-burn signal. However, there is no obvious differences between no-burn and burn signals within the higher frequency bandwidth. Hence, new method should be proposed to distinguish the grinding burn.

\subsection{Signal Analysis by XWT and WTC}

From the experiment result, it is known that when grinding burn happens, the $\mathrm{AE}$ signal induced by metal burn is 


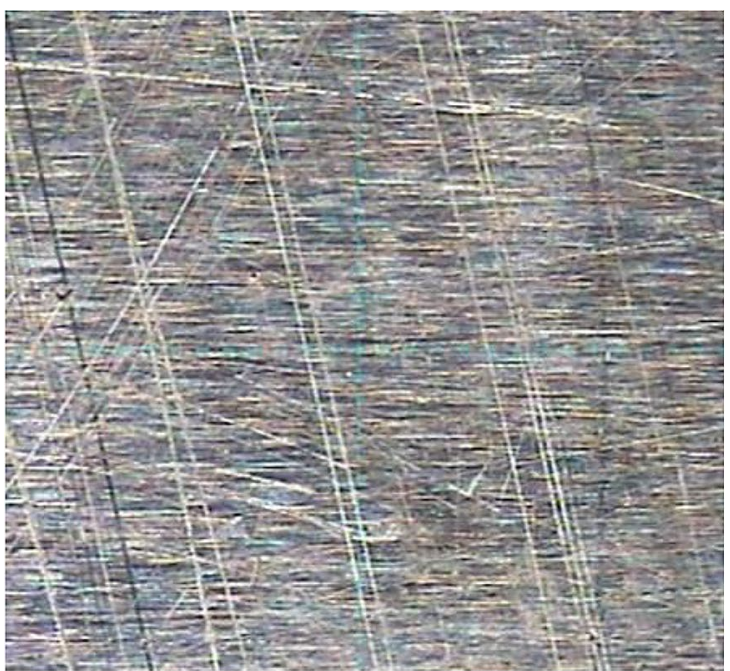

a No-burn surface by laser

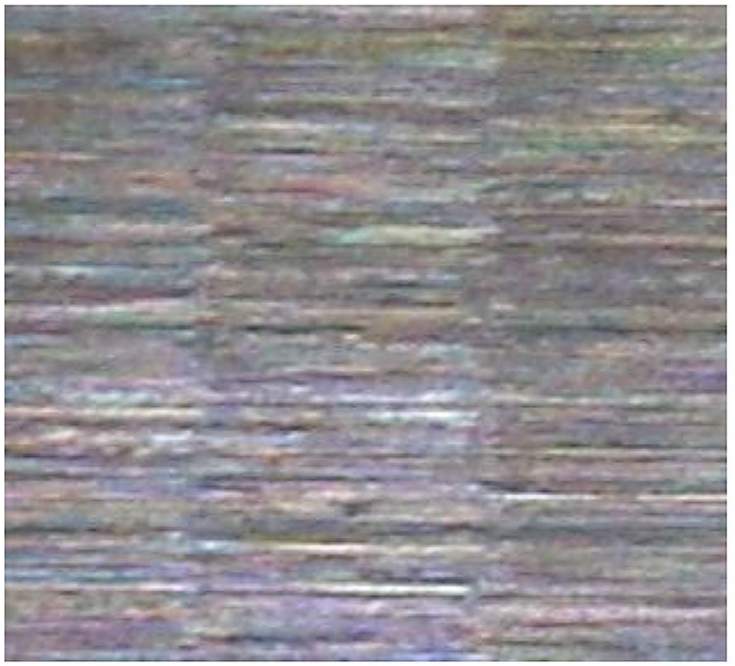

c No-burn surface by grinding

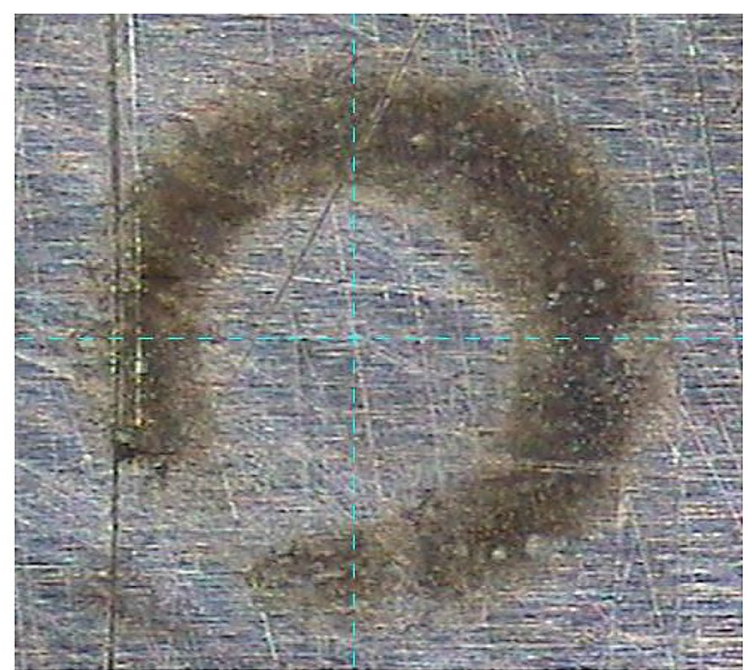

b Burn surface by laser

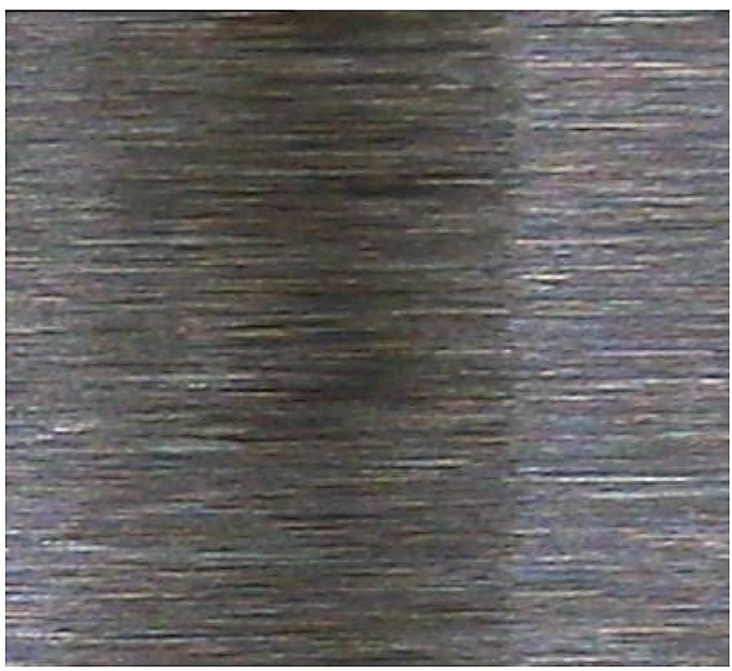

d Burn surface by grinding

Figure 3 Optical image of laser heating surfaces and grinding surfaces

easily covered by a variety of $\mathrm{AE}$ resources induced during grinding process. And sometimes it is hard to distinguish no-burn and burn signals only by RMS value. Therefore, obtaining and monitoring the frequency components which indicate the occurrence of metal burn can improve the detection accuracy of grinding burn. In order to extract the features which represent the grinding burn signals, the XWT and WTC are employed in the paper.

It is known that the XWT reveals the common areas with high power and WTC exposes co-vary character of two signals in time-frequency space. Statistically speaking, the coherence of two signals increases with the WTC value.
In order to obtain XWT and WTC results, the wavelet transform must be adopted first. According to Eq. (1), the wavelet transform of laser heating $\mathrm{AE}$ signal is denoted by $W_{x}(u, s)$. The wavelet transform of grinding $\mathrm{AE}$ signal is denoted by $W_{y}(u, s)$. Then the XWT and WTC of the two kinds of AE signals can be obtained by Eq. (3) and Eq. (4). Figure 6 shows the CWT results of no-burn and burn signals produced by laser. The energy of signal is normalized. The peak frequency of metal burn signal centers around $310 \mathrm{kHz}$. Figure 7 presents the CWT results of no-burn and burn signals of grinding process. The energy of signal is also normalized. The main energy of grinding signals focuses below $100 \mathrm{kHz}$. The energy 

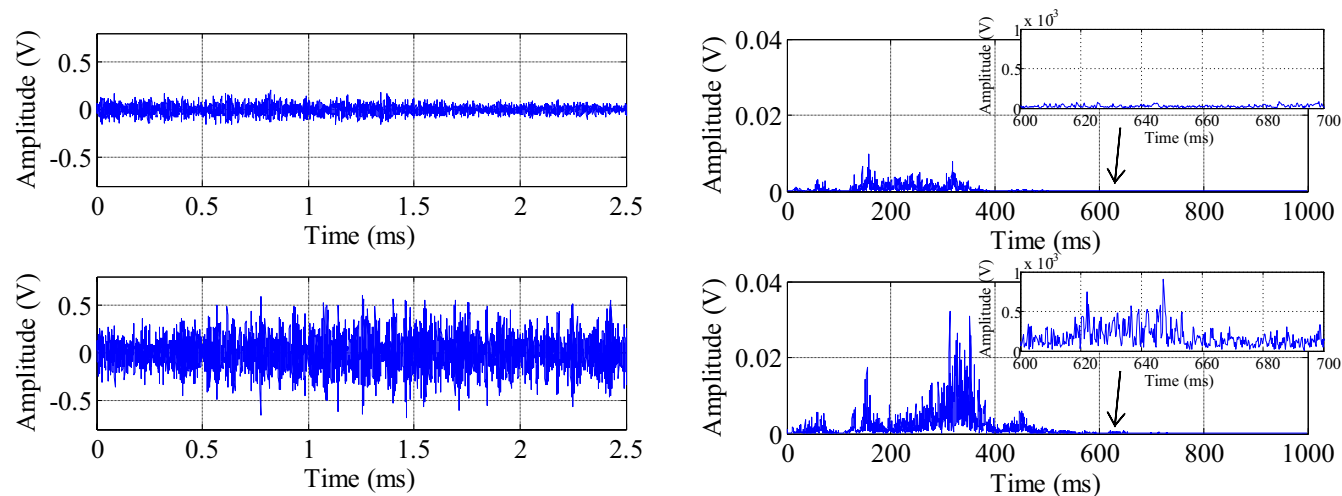

a Waveforms of no-burn and burn signal by laser

b Spectrums of no-burn and burn signal by laser

Figure 4 AE signals produced by laser heating
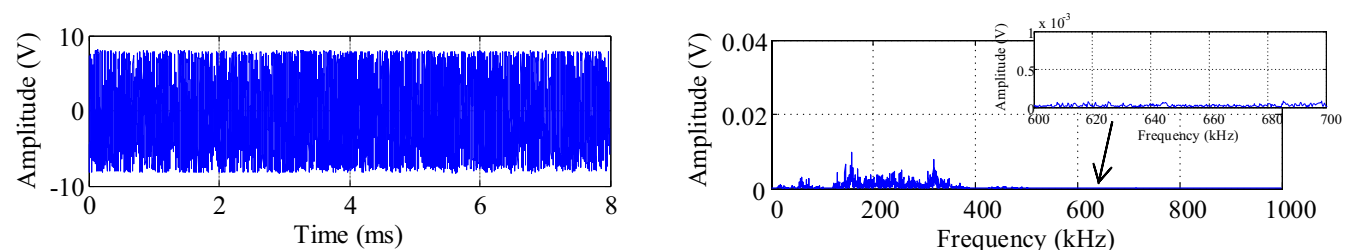

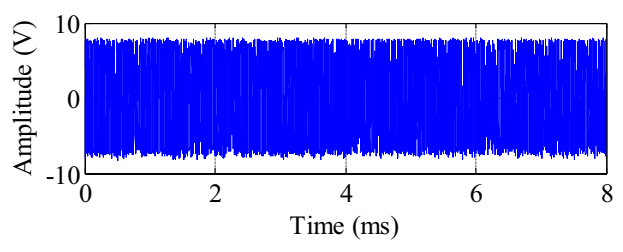

a Waveforms of no-burn and burn grinding signal

Figure 5 AE signals produced by grinding process

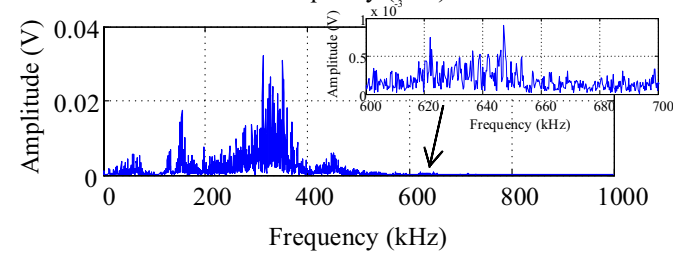

b Spectrums of no-burn and burn grinding signal

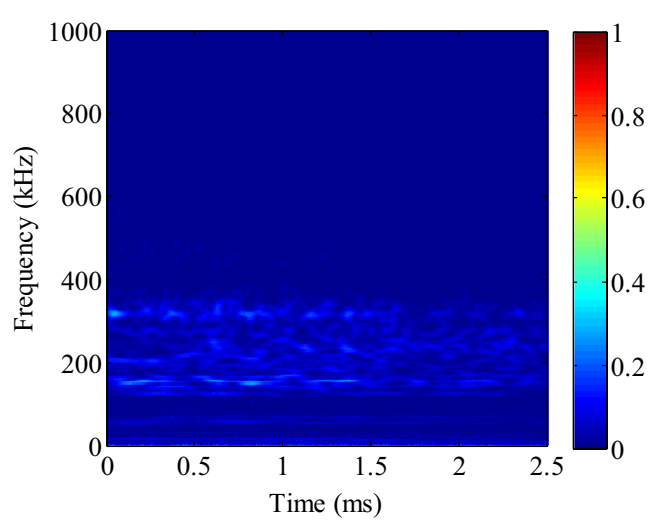

a CWT of no-burn signal by laser heating

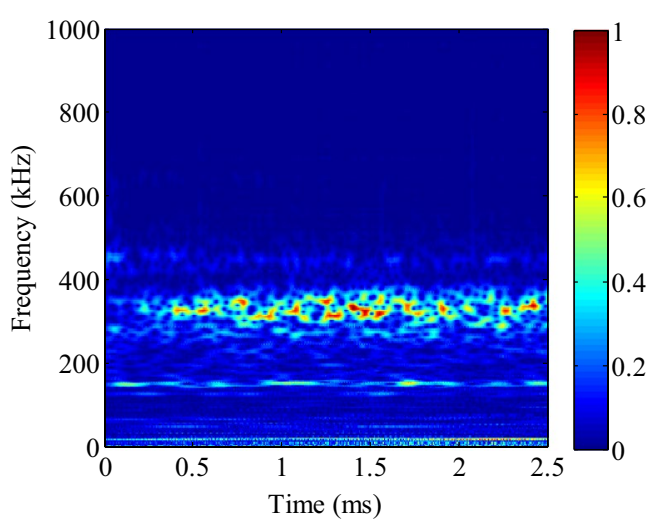

b CWT of burn signal by laser heating

Figure 6 AE signals produced by laser heating 
above $100 \mathrm{kHz}$ is relatively low no matter grinding burn happens or not.

Figure 8 is the XWT of laser and grinding signals. Figure $8(\mathrm{a})$ is the XWT of laser burn signal and grinding burn signal. Figure $8(\mathrm{~b})$ is the XWT of laser burn signal and normal grinding signal. Figure $8(\mathrm{c})$ is the XWT of no-burn signal and grinding burn signal. Figure 8(d) is the XWT of no-burn signal and normal grinding signal. The energy around $310 \mathrm{kHz}$ in Figure $8(\mathrm{a})$ is higher than other frequency bands. Comparing with the CWT results, the frequency components of metal burn in the grinding burn signal are enhanced by XWT. The background noise of grinding process is weakened accordingly. In Figure 8(c) and Figure 8(d), the energy around $310 \mathrm{kHz}$ is much lower than Figure $8(\mathrm{a})$ and Figure $8(\mathrm{~b})$. In Figure 8(a) and Figure 8(b), common power areas also appear below $100 \mathrm{kHz}$. From the metal burn experiment, it is known that the metal burn AE signals distributes within $250 \mathrm{kHz}$ to $650 \mathrm{kHz}$. Thus components below $100 \mathrm{kHz}$ is beyond our consideration. Therefore, differences between Figure 8(a) and Figure 8(b) around $310 \mathrm{kHz}$ are utilized to distinguish the grinding burn in the following section.

Figure 9(a) depicts the WTC result of the laser burn signal and grinding burn signal. Figure 9(b) depicts the WTC result of the laser burn signal and normal grinding signal. Figure $9(\mathrm{c})$ is the WTC result of laser no-burn and grinding burn signal. Figure $9(\mathrm{~d})$ is the WTC result of laser no-burn and normal grinding signal. It shows that the high correlation coefficient focuses between $200 \mathrm{kHz}$ to $530 \mathrm{kHz}$. Metal burn AE signal and grinding burn AE signal have low coherence relationship below $100 \mathrm{kHz}$. Between $200 \mathrm{kHz}$ to $400 \mathrm{kHz}$, the two kinds of AE burn signals have the highest coherence relationship. But it is hard to distinguish grinding burn by WTC within this frequency band. Fortunately, between $600 \mathrm{kHz}$ to $650 \mathrm{kHz}$, there is successive correlation frequency between laser burn and grinding burn signal. Considering the result in Figure 5(b), the background noise of this bandwidth is much lower than other frequency bands. It indicates that within this bandwidth, most energy of the grinding burn $\mathrm{AE}$ signal is originated from the metal burn signal. Therefore, by comparing the frequency difference within $600 \mathrm{kHz}$ to $650 \mathrm{kHz}$, the grinding burn can be distinguished. Therefore, both the XWT and WTC results can be used to indicate occurrence of grinding burn.

The metal burn AE signal is transient and time-varying. And the AE burn signals of the two experiments are collected independently. Therefore, the phase correlation between the signals is meaningless here.

Comparing with EEMD, the computing time of XWT and WTC of signals length of 8192 are $3.13 \mathrm{~s}$ and $3.88 \mathrm{~s}$. The computing time of the same length signal by EEMD is $22.28 \mathrm{~s}$. Therefore, the proposed method in the paper is more suitable for online monitoring system.

\subsection{Detection of Grinding Burn}

In order to extract effective information, energy of XWT (EXWT) and degree of WTC (DWTC) are defined. The EXWT is defined as the sum of XWT of pure metal burn signal and grinding signal of unknown status within certain frequency band and time interval, see in Eq. (8):

$$
\text { EXWT }=\sum_{0}^{T} \sum_{f_{1}}^{f_{2}}\left|W_{x_{2}, y_{\text {unknown }}}(u, s)\right|,
$$

where $f_{1}$ and $f_{2}$ are the lower and upper limit of frequency band.

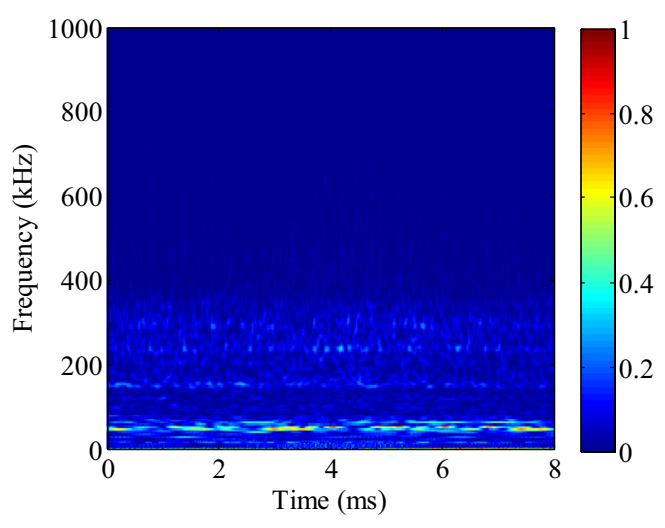

a CWT of no-burn signal by grinding process

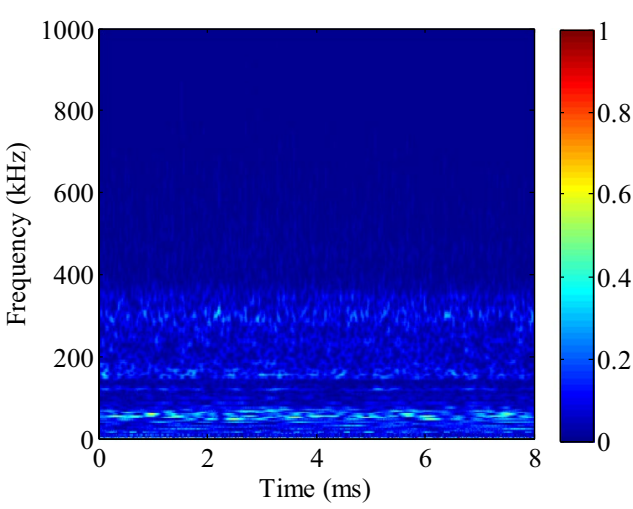

b CWT of burn signal by grinding process

Figure 7 AE signals produced by grinding process 


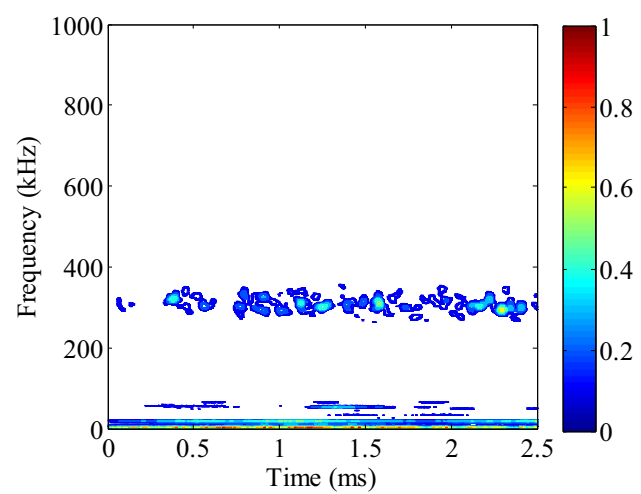

a Laser burn vs grinding burn signal

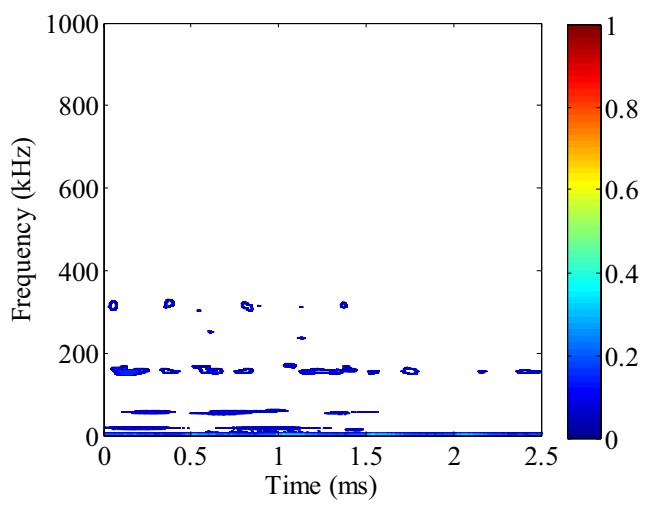

c Laser no-burn vs grinding burn signal

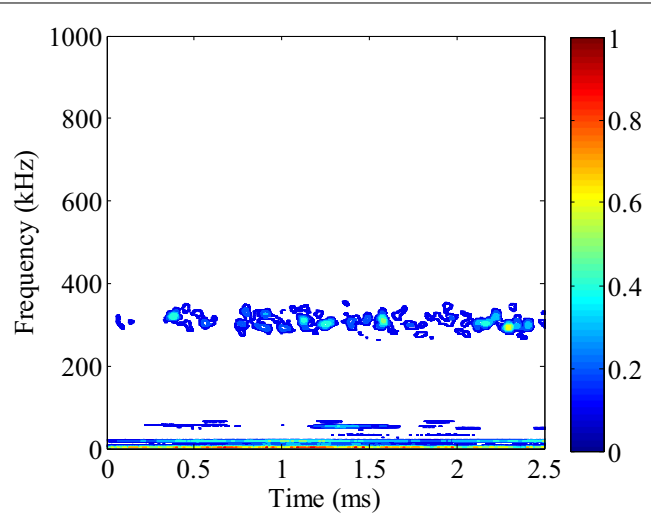

b Laser burn vs normal grinding signal

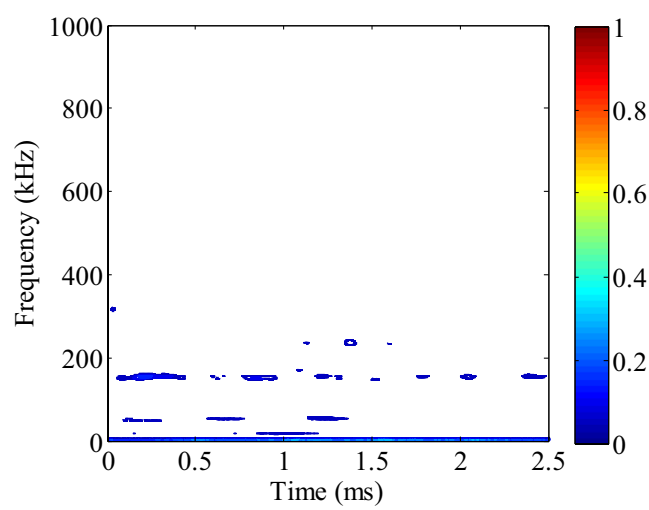

d Laser no-burn vs normal grinding signal

Figure 8 XWT of laser and grinding signals

The definition of DWTC is as follows:

DWTC $=\frac{\left[\sum_{0}^{T} \sum_{f_{1}}^{f_{2}}\left(\mathrm{WTC}_{x_{2}, y_{\text {unknown }}}-\overline{\left.\sum_{0}^{T} \sum_{f_{1}}^{f_{2}} \mathrm{WTC}_{x_{2}, y_{1}}\right)}\right]^{4} / T\right.}{\sigma_{\text {WTC }_{x_{2}, y_{1}} f_{f_{1}}^{4}}^{4}}$,

where $\sigma^{2}$ is variance.

According to the results of the previous sections, the frequency band of EXWT is set to $300 \mathrm{kHz}$ to $340 \mathrm{kHz}$ and the DWTC is $600 \mathrm{kHz}$ to $650 \mathrm{kHz}$. Sixty sets of normal grinding signals and grinding burn signals are employed. The two indexes are calculated to classify the grinding burn signals. The classification result is shown in Figure 10. The two sets have clearly boundaries which can be distinguished without doubt. The grinding burn detection accuracy achieves $100 \%$.

\section{Conclusions}

In this paper, a new grinding burn detection method is proposed. The metal burn and grinding burn experiments are carried out by which the AE signals are obtained. The relationship between $\mathrm{AE}$ signals induced by pure metal burn and grinding burn process is established by XWT and WTC methods. Novel index, EXWT and DWTC are calculated to distinguish grinding burn signals, by which the accuracy of grinding burn classification achieves $100 \%$. From the research work, the following conclusions can be made.

1. The metal burn AE signals and grinding burn $\mathrm{AE}$ signals have common high energy area within frequency band of $250 \mathrm{kHz}$ to $400 \mathrm{kHz}$. They also have co-vary character within frequency band of $250 \mathrm{kHz}$ to $650 \mathrm{kHz}$.

2. The relationship of metal burn and grinding burn signals can be found by XWT and WTC method. The common area with high energy of two kinds of $\mathrm{AE}$ signals can be enhanced by XWT. The coherence relationship of two kinds of signals can be found by WTC. Both of them can eliminate the unwanted AE sources and background noises to some extent.

3. New parameters are proposed. EXWT and DWTC within certain frequency bandwidth are capable to detect grinding burn accurately. 


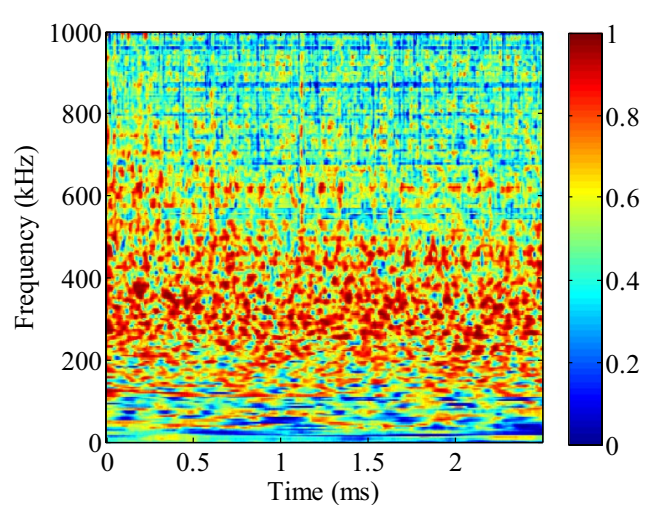

a Laser burn vs grinding burn

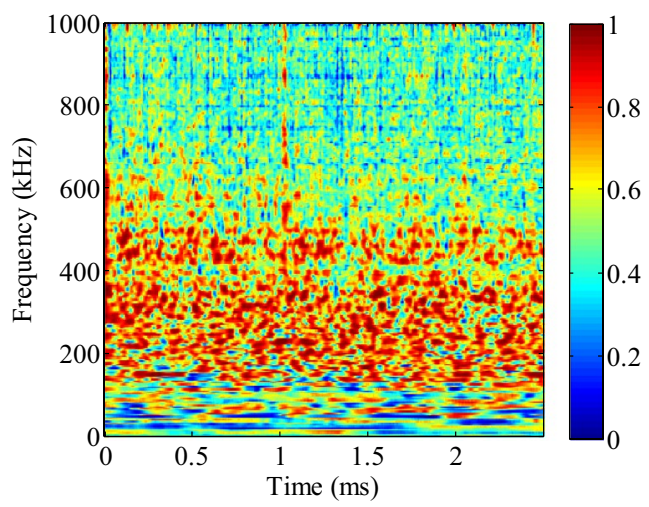

c Laser no-burn vs grinding burn signal

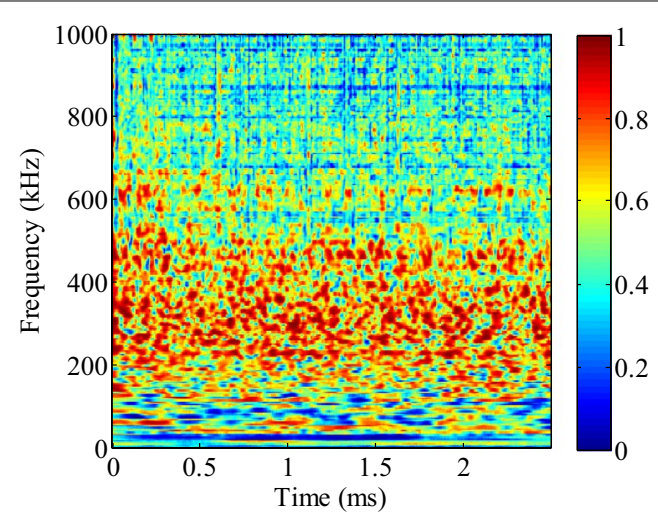

b Laser burn vs normal grinding signal

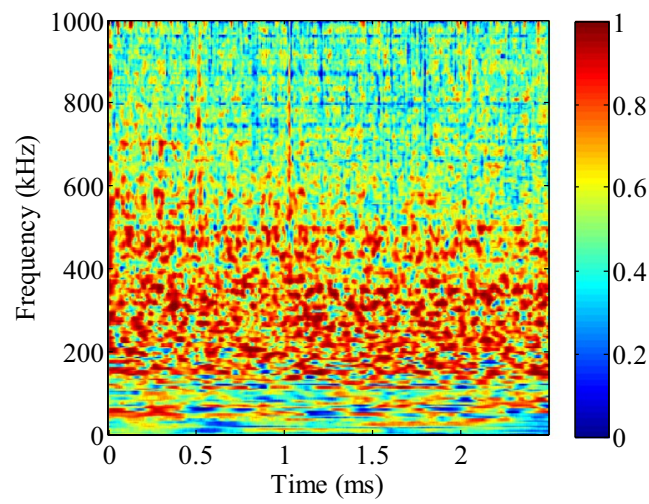

d Laser no-burn vs normal grinding signal

Figure 9 WTC result of the laser signal and grinding signal

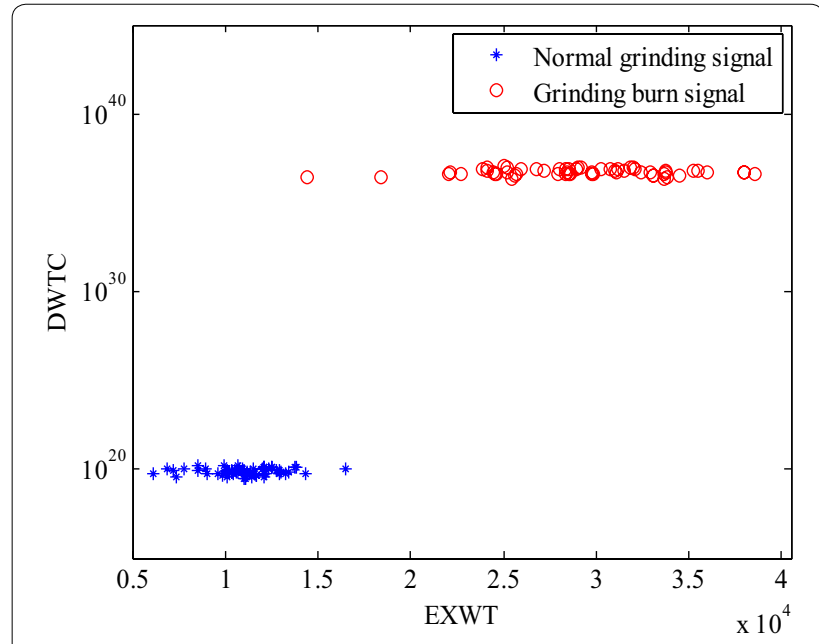

Figure 10 Classification by EXWT and DWTC

\section{Authors' Contributions}

ZG was in charge of the whole trial. ZG analyzed the data and wrote the manuscript. JL gave final approval of the version to be published. XW assisted with sampling and laboratory analyses. YL revised the manuscript. All authors read and approved the final manuscript.

\section{Authors' Information}

Zheyu Gao, is a PhD candidate at School of Mechanical Engineering in Xi'an Jiaotong University. She received her B.S. and M.S. degree in Energy and Power Engineering Institute in Xi'an Jiaotong University. Her research interests include Acoustic Emission technology, signal processing, quality monitoring and fault diagnosis.

Jing Lin, is a professor at School of Reliability and Systems Engineering, Beihang University. He obtained his B.S., M.S. and PhD degrees from School of Mechanical Engineering, Xi'an Jiaotong University. He was working as a postdoctoral fellow at University of Alberta, Canada. He obtained the National Science Fund for Distinguished Young Scholars in 2011 and Cheung Kong Scholars in 2017. Now his research directions are processing quality monitoring, mechanical system reliability, fault diagnosis and signal processing.

Xiufeng Wang, is a lecture at School of Mechanical Engineering, Xi'an Jiaotong University. He received his B.S., M.S. and PhD degrees from School of Mechanical Engineering, Xi'an Jiaotong University. He was working as a visiting scholar in University of Bristol, England. His research interests include fault diagnosis of rotating machinery, mechanical signal processing and rotor dynamics.

Yehe Liao, is a lecture at School of Mechanical Engineering, Xi'an Jiaotong University. He received his M.S. and PhD degrees from School of Mechanical Engineering, Xi'an Jiaotong University. He is working in Research Institute of Diagnostics and Cybernetics, Xi'an Jiaotong University. His research interests include fault diagnosis of mechanical system, intelligent diagnosis and rotor dynamics.

\section{Competing Interests}

The authors declare that they have no competing interests. 


\section{Funding}

Supported by National Natural Science Foundation of China (Grant No. 51421004), and Fundamental Research Funds of the Central Universities of China (Grant No. CXTD2014001).

\section{Author Details}

1 Shaanxi Key Laboratory of Mechanical Product Quality Assurance and Diagnostics, Xi'an Jiaotong University, Xi'an 710049, China. ${ }^{2}$ School of Reliability and Systems Engineering, Beihang University, Beijing 100191, China.

Received: 12 December 2018 Revised: 3 July 2019 Accepted: 7 August 2019

Published online: 19 August 2019

\section{References}

[1] S Malkin. Grinding technology-theory and applications of machining with abrasives. China: NEU Press, 2002. (in Chinese)

[2] B P Gu, Z S Yang, P Long. Numerical modeling and experimental study of microcrack detection in grinding burn using high order strain mode. International Journal of Advanced Manufacturing Technology, 2015, 80(5-8): 859-870.

[3] A Thanedar, G G Dongre, S S Joshi. Analytical modelling of temperature in cylindrical grinding to predict grinding burns. International Journal of Precision Engineering and Manufacturing, 2019, 20(1): 13-25.

[4] B Lin, K Zhou, J Guo, et al. Influence of grinding parameters on surface temperature and burn behaviors of grinding rail. Tribology International, 2018, 122: 151-162.

[5] L Y Li, L J Sun, Y Zhao, et al. Control technology of raceway grinding burn of high-temperature steel bearing rings. Failure Analysis \& Prevention, 2017, 12(6): 349-353. (in Chinese)

[6] G Rf Neto, M Marchi, C Martins, et al. Monitoring of grinding burn by AE and vibration signals. Proceedings of the 6 th International Conference on Agents and Artificial Intelligence, Angers, France, Mar 6-8, 2014, 1: 272-279.

[7] PR Aguiar, E C Bianchi, R C Canarim. Monitoring of grinding burn by Acoustic Emission. Acoustic Emission. Brazil, InTech, 2012

[8] X Q Zhang, L S Chen, H Li, et al. Study on grinding burn in high efficiency deep grinding. Applied Mechanics and Materials, 2013, 389: 318-323.

[9] D M S Ribeiro, P R Aguiar, L F G Fabiano, et al. Spectra measurements using piezoelectric diaphragms to detect burn in grinding process. IEEE Transactions on Instrumentation and Measurement, 2017, 66: 3052-3062.

[10] P R Aguiar, P J A Serni, E C Bianchi, et al. In-process grinding monitoring by acoustic emission. IEEE International Conference on Acoustics, Speech, and Signal Processing. IEEE, Montreal, Canada, May 17-21, 2004: 389-405.

[11] M Neslušan, J Čížek, K Kolařík, et al. Monitoring of grinding burn via Barkhausen noise emission in case-hardened steel in large-bearing production. Journal of Materials Processing Technology, 2017, 240: 104-117.

[12] R Ito, N Mukaide, T Azuma, et al. Development of non-destructive inspection system for grinding burn-in-process detection of grinding burn. Advanced Materials Research, 2014, 1017: 135-140.

[13] B F He, C E Wei, ZY Shi. Researching status and developing direction of gear grinding burn detection methods. Chinese Journal of Scientific Instrument, 2017, 38(8): 1889-1900. (in Chinese)

[14] W Long, XTian, L Qian, et al. Grinding burn evaluation for 20CrMnTi steel based on binary images and neural network. International Journal of Advanced Manufacturing Technology, 2017, 93(4): 1-10.

[15] C H Lauro, L C Brandao, D Baldo, et al. Monitoring and processing signal applied in machining processes - A review. Measurement, 2014, 58: 73-86.

[16] J S Kwak, M K Ha. Neural network approach for diagnosis of grinding operation by acoustic emission and power signals. Journal of Materials Processing Technology, 2004, 147: 65-71.

[17] K Jemielnial, J Kossakowska. Tool wear monitoring based on wavelet transform of raw acoustic emission signal. Advance Manufacture of Science Technology, 2010, 34: 5-17.

[18] A Bell, T Jin, D J Stephenson. Burn threshold prediction for High Efficiency Deep Grinding. International Journal of Machine Tools \& Manufacture, 2011, 51: 433-438
[19] J M Griffin. Traceability of acoustic emission measurements for micro and macro grinding phenomena-characteristics and identification through classification of micro mechanics with regression to burn using signal analysis. International Journal of Advanced Manufacturing Technology, 2015, 81: 1463-1474.

[20] Q Liu, X Chen, G Nabil. Investigation of acoustic emission signals under a simulative environment of grinding burn. International Journal of Machine Tools \& Manufacture, 2006, 46: 284-292.

[21] Z S Yang, HXWu, Z HYu, et al. A non-destructive surface burn detection method for ferrous metals based on acoustic emission and ensemble empirical mode decomposition: from laser simulation to grinding process. Measurement Science and Technology, 2014, 25: 035602.

[22] A Mohammed, J Folks, X Chen. Detection of grinding temperatures using laser irradiation and acoustic emission sensing technique. Materials and Manufacturing Processes, 2012, 27: 395-400.

[23] P RWu. A study of grinding burn monitoring using multi-sensor fusion. Taiwan, China: Chung Hsing University, 2015. (in Chinese)

[24] Z S Yang, Z H Yu, C Xie, et al. Application of Hilbert-Huang transform to acoustic emission signal for burn feature extraction in surface grinding process. Measurement, 2014, 47: 14-21.

[25] Q Liu, X Chen, N Gindy. Fuzzy pattern recognition of AE signals for grinding burn. International Journal of Machine Tools \& Manufacture, 2005, 45: 811-818.

[26] Z Wang, P Willett, P R Aguiar, et al. Neural network detection of grinding burn from acoustic emission. International Journal of Machine Tools \& Manufacture, 2001, 41(2): 283-309.

[27] Z H Yang, Z H Yu. Experimental study of burn classification and prediction using indirect method in surface grinding of AISI 1045 steel. International Journal of Advanced Manufacturing Technology, 2013, 68: 2439-2449.

[28] P Lajmert, B Kruszynski. A diagnostic system for cylindrical plunge grinding process based on Hilbert-Huang transform and principal component analysis. Advances in Manufacturing Science and Technology, 2010, 34 : 19-30.

[29] A Saravanapriyan, L Vijayaraghavan, R Krishnamurthy. On-line detection of grinding burn by integrated sensing. Proceedings of the First ISA/IEEE Conference, Rosemont, USA, November 5-7, 2001: 89-94.

[30] CTorrence, G P Compo. A practical guide to wavelet analysis. Bulletin of The American Meteorological Society, 1998, 79(1): 61-78.

[31] A Grinsted, J C Moore, S Jevrejeva. Application of the cross wavelet transform and wavelet coherence to geophysical time series. Nonlinear Processes in Geophysics, 2004, 11: 561-566.

[32] J P Lachaux, A Lutz, D Rudrauf, et al. Estimating the time-course of coherence between single-trial brain signals: an introduction to wavelet coherence. Neurophysiologie Clinique Clinical Neurophysiology, 2002, 32: 157-174.

[33] A Klein, T Sauer, A Jedynak, et al. Conventional and wavelet coherence applied to sensory-evoked electrical brain activity. IEEE Transactions on Biomedical Engineering, 2006, 53(2): 266-272.

[34] Y Liu, X F Wang, J Lin, et al. Correlation analysis of motor current and chatter vibration in grinding using complex continuous wavelet coherence. Measurement Science and Technology, 2016, 27(11): 115106.

[35] J Lin, L S Qu. Feature extraction based on Morlet wavelet and its application for mechanical fault diagnosis. Journal of Sound and Vibration, 2000, 234(1): 135-148

[36] I Daubechies. Ten lectures on wavelets. Beijing: National Defence Industry Press, 2011. (in Chinese)

[37] X M Zhang, YY He, et al. Parameters optimization of continuous wavelet transform and its application in Acoustic Emission signal analysis of rolling bearing. Chinese Journal of Mechanical Engineering, 2007, 20(2): 104-108.

[38] S Mallat. A wavelet tour of signal processing-the sparse way. Beijing: China Machine Press, 2011. (in Chinese) 